\title{
QCD PHASE DIAGRAM IN NONLOCAL CHIRAL QUARK MODELS
}

\author{
D. GÓMEZ DUMM \\ IFLP, CONICET - Depto. de Física, Univ. Nac. de La Plata, \\ C.C. 67, 1900 La Plata, Argentina \\ E-mail:dumm@fisica.unlp.edu.ar
}

\begin{abstract}
The QCD phase diagram is analyzed within chiral quark models with nonlocal separable interactions. For the case of two light flavors, we describe in detail the characteristics of the chiral phase transition in the $T-\mu$ plane, and analyze the color superconducting phase transition taking place at relatively large chemical potentials. We also consider the three-flavor version of the models, analyzing the predictions for masses and decay constants of light pseudoscalar mesons.
\end{abstract}

\section{Introduction}

The behavior of strongly interacting matter at finite temperature and/or density is a subject of fundamental interest, with applications in cosmology, astrophysics, and in the physics of relativistic heavy ion collisions. In view of the difficulty found when dealing with strong interactions in the nonperturbative regime, it has not been possible yet to obtain detailed information about the full $T-\mu$ phase diagram directly from QCD, and most of the theoretical work on the subject relies in low energy effective models. One of the most popular ones is that proposed long ago by Nambu and Jona-Lasinio (NJL) ${ }^{1}$, in which quarks interact through local four fermion couplings. In the last few years, covariant nonlocal extensions of this model have been proposed ${ }^{2}$, mainly motivated by some approaches to low energy quark dynamics (instanton liquid model, Schwinger-Dyson resummation techniques) in which nonlocality arises in a natural way. It has also been shown that these nonlocal theories present several advantages in comparison with the standard NJL scheme. Here we analyze, in this context, the structure of the QCD phase diagram. First, we consider the case of twoflavor models, studying the features of the chiral phase transition for finite $T$ and $\mu$ and the presence of a possible superconducting phase in the region of large $\mu$. Then we also address the three-flavor case, studying the 
predicted pattern of pseudoscalar meson masses and decay constants. The analysis summarized here is developed in detail in Refs. ${ }^{3,4,5,6}$.

\section{Formulation}

We start by quoting the Euclidean quark effective action

$S_{E}=\int d^{4} x\left\{\bar{\psi}(x)\left(-i \not \partial+m_{c}\right) \psi(x)-\frac{G}{2}\left[j^{S}(x) j^{S}(x)+j_{k}^{P}(x) j_{k}^{P}(x)\right]\right\}$,

where $\psi \equiv(u d)^{T}$, while the fermion currents $j^{S}(x)$ and $j_{k}^{P}(x)$ are given by

$$
\left\{\begin{array}{l}
j^{S}(x) \\
j_{k}^{P}(x)
\end{array}\right\}=\int d^{4} y d^{4} z r(y-x) r(x-z) \bar{\psi}(y)\left\{\begin{array}{c}
\mathbb{1} \\
i \gamma_{5} \tau_{k}
\end{array}\right\} \psi(z) .
$$

Here $r(x-y)$ is a soft covariant nonlocal regulator, which in the case of the instanton liquid model has a definite form in terms of modified Bessel functions. In the following we will consider the relatively simple case of a Gaussian regulator $r\left(p^{2}\right)=\exp \left(-p^{2} / 2 \Lambda^{2}\right)$, where $\Lambda$ is a free parameter of the model, playing the rôle of an ultraviolet cutoff scale. In any case, our results are found to be qualitatively similar for other regulator shapes. Notice that the constituent quark mass $m_{c}$ (we assume isospin symmetry) and the coupling constant $G$ are also free parameters of the model.

We perform now a bosonization of the partition function of the theory, $Z=\int D \bar{\psi} D \psi \exp \left(-S_{E}\right)$. To do this we introduce scalar and pseudoscalar meson fields $\sigma(x), \pi_{k}(x)$, integrating out the quark degrees of freedom. Then we carry out the mean field approximation (MFA), in which the meson fields are expanded around their translational invariant vacuum expectation values $\bar{\sigma}$ and $\bar{\pi}_{k}$ (the latter vanish due to charge and isospin symmetries). The extension to finite $T$ and $\mu$ is obtained by performing the replacement

$$
\int \frac{d^{4} p}{(2 \pi)^{4}} F\left(p_{4}, \vec{p}\right) \rightarrow \bigvee_{p} F\left(p_{4}, \vec{p}\right) \equiv T \sum_{n=-\infty}^{\infty} \int \frac{d^{3} p}{(2 \pi)^{3}} F\left(\omega_{n}-i \mu, \vec{p}\right)
$$

in the four-momentum integrals arising from the fermion determinant (here $\omega_{n}$ are Matsubara frequencies corresponding to fermion modes). The grand canonical thermodinamical potential per unit volume is then given by

$$
\omega_{\mathrm{MFA}}\left(T, \mu, m_{c}\right)=-\frac{T}{V} \log Z_{\mathrm{MFA}}=\frac{\bar{\sigma}^{2}}{2 G}-4 N_{c} \bigvee_{p} \log \left[p^{2}+\Sigma^{2}\left(p^{2}\right)\right],
$$

where $\Sigma\left(p^{2}\right)=m_{c}+\bar{\sigma} r^{2}\left(p^{2}\right)$ is the constituent (momentum-dependent) quark mass. Now $\bar{\sigma}\left(T, \mu, m_{c}\right)$ can be obtained from $\partial \omega_{\mathrm{MFA}} / \partial \bar{\sigma}=0$ (gap 
equation), and one can easily derive the expressions for other relevant physical quantities, such as the quark condensate $\langle\bar{q} q\rangle$, quark density $\rho$, chiral susceptibility $\chi_{V, T, \mu}=-\left(\partial\langle\bar{q} q\rangle / \partial m_{c}\right)_{T, \mu}$, pressure, energy density, etc.

\section{Chiral phase transition: analytical and numerical analysis}

In order to study the physical quantities, the Matsubara sums can be worked out using complex plane analysis. While in the standard (local) NJL model the Euclidean quark propagator has just two simple poles at $p_{4}= \pm i \sqrt{\vec{p}^{2}+M^{2}}$, being $M$ the quark constituent mass, in the case of nonlocal models the regulator leads in general to a complicated structure of poles and cuts in the complex plane. Depending on the parameters of the model and the shape of the regulator, there may be some poles on the real axis or not; it has been suggested that this last situation is a sort of realization of confinement, in the sense that the effective quark propagator has no poles at real energies ${ }^{7}$, and one can talk about "confining" and "nonconfining" parameter sets. From our analysis it is seen ${ }^{3,4}$ that using adequate integration paths, and introducing some auxiliary complex occupation number functions, the Matsubara sums can be converted into integrals. Moreover, we have found that in all considered cases the behavior of the physical quantities is strongly dominated by the contribution of the first pole of the quark propagator.

We have carried out numerical calculations to evaluate the dependence of the mentioned physical quantities with $T$ and $\mu$. To proceed, we have adjusted the free parameters $\Lambda, m_{c}$ and $G$ so as to reproduce the empirical values of the pion mass and decay constant, whereas as the third input we have chosen some fixed values of $\Sigma(0)$. Both confining and nonconfining parameter sets have been considered, studying in each case the features of the chiral phase transition. In general, the results have been found to be qualitatively similar (a detailed comparison can be found in Refs. ${ }^{3,4}$ ); for definiteness we present here the curves corresponding to one of these parameter sets, namely $\Lambda=760 \mathrm{MeV}, m_{c}=7.7 \mathrm{MeV}$ and $G=30 \mathrm{GeV}^{-2}$.

Our main results are shown in Fig. 1, where we quote the values of the quark condensate, the density (relative to nuclear matter density $\rho_{0}$ ) and the chiral susceptibility as functions of $T$ and $\mu$. By looking at the curves for the quark condensate, it is seen that for $T=0$ there is a first order phase transition at a given critical chemical potential $\mu_{c}$. The latter goes down as $T$ is increased, until one reaches an end point where the first order phase transition turns into a smooth crossover. For this parameter set the 

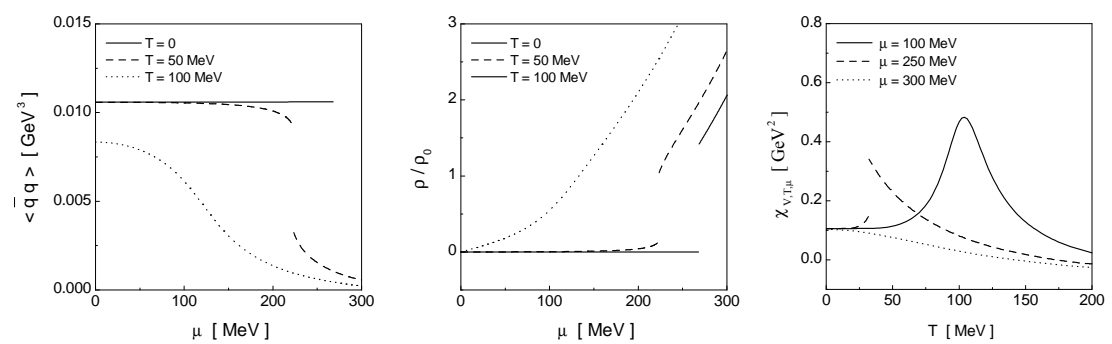

Figure 1. Behavior of the chiral condensate (left), the density (center) and the chiral susceptibility (right) with the temperature and the chemical potential.

end point is located at $\left(T_{E}, \mu_{E}\right)=(55 \mathrm{MeV}, 210 \mathrm{MeV})$. In the crossover region, the transition is also well defined by the presence of marked peaks in the chiral susceptibility, the sharpness of these peaks giving a measure of the crossover steepness. Thus we arrive at the phase diagrams shown in Fig. 2, in which we plot the phase transition temperature as a function of the chemical potential (left) and the density (right). The latter includes a "mixed phase" zone, which can be interpreted as a region where droplets containing light quarks of mass $m_{c}$ coexist with a gas of constituent, massive quarks. It should be noticed that the model predicts a critical temperature at $\mu=0$ of about $100 \mathrm{MeV}$, somewhat below the results obtained from lattice calculations which suggest this value to be $\sim 140-190 \mathrm{MeV}$.
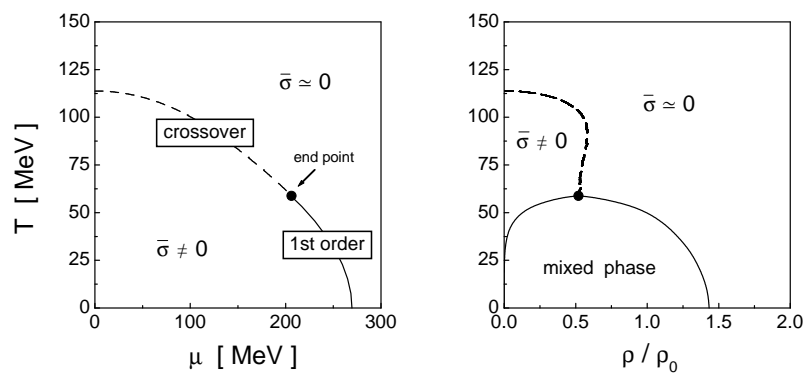

Figure 2. Phase transition temperature as a function of the chemical potential (left) and the density (right). Solid (dashed) lines stand for first order transition (crossover).

\section{Extensions to diquark couplings and three flavors}

The ability of this kind of models to allow for a two-flavor superconducting (2SC) phase at large chemical potentials is also under study ${ }^{5}$. This analysis 
considers an effective quark-quark interaction in the Euclidean action (1), carrying a new coupling constant $h$. Preliminary results show that for low $T$ a first order phase transition into a $2 \mathrm{SC}$ phase is expected at some critical value of $\mu$. A similar behavior is found when $T$ is increased, until one reaches a "triple point" in which all three phases (hadronic, chiral and 2SC) coexist. The phase diagram is then completed with the inclusion of a second order phase transition line between the chiral and the 2SC phases. Unfortunately, the precise features of the phase diagram depend on the value of the diquark coupling $h$, which cannot be fixed from low energy phenomenology. A detailed discussion in this sense is given in Ref. ${ }^{5}$.

Finally, we have considered ${ }^{6}$ the inclusion of strange degrees of freedom, extending the flavor symmetry of the model to $S U(3)$ and incorporating the the $U(1)_{A}$ symmetry breaking through a nonlocal 't Hooft-like coupling. As a first step we have concentrated on the evaluation of meson masses and decay constants, finding our results in quite good agreement with empirical values, in particular in the case of the ratio $f_{K} / f_{\pi}$ and the anomalous decay $\pi^{0} \rightarrow \gamma \gamma$ (which are problematic in the standard NJL model). We have also obtained a reasonable description of the $\eta-\eta^{\prime}$ phenomenology. Remarkably, this requires the presence of two significantly different state mixing angles.

Clearly, the two-flavor diquark channel analysis cannot be trusted beyond the strange quark mass threshold, and the $S U(3)$ version of the model should be addressed, leading presumably to the presence of a color flavor locking phase. We expect to report on these issues in forthcoming works.

\section{Acknowledgments}

I am indebted to N.N. Scoccola and A. Grunfeld for useful discussions, and to the organizers of SEWM04 for financial aid. This work has been partially supported by ANPCyT (Argentina) under grant PICT02-03-10718.

\section{References}

1. Y. Nambu and G. Jona-Lasinio, Phys. Rev. 122, 345 (1961); Phys. Rev. 124, 246 (1961). See also S. Klevansky, Rev. Mod. Phys. 64, 649 (1992).

2. G. Ripka, Quarks bound by chiral fields (Oxford Univ. Press, Oxford, 1997).

3. I. General, D. Gómez Dumm and N.N. Scoccola, Phys. Lett. B506, 267 (2001).

4. D. Gómez Dumm and N.N. Scoccola, Phys. Rev. D65, 074021 (2002).

5. R.S. Duhau, A.G. Grunfeld and N.N. Scoccola, hep-ph/0409072.

6. A. Scarpettini, D. Gómez Dumm and N.N. Scoccola, Phys. Rev. D69, 114018 (2004).

7. R.D. Bowler and M.C. Birse, Nucl. Phys. A582, 655 (1995). 